\title{
Investigation of the Quadrupole Moment and Form Factors of Some Ca Isotopes
}

\begin{abstract}
Ahmed H. Ali
Received 3/7/2019, Accepted 24/9/2019, Published 1/6/2020

This work is licensed under a Creative Commons Attribution 4.0 International License.

Abstract:

Nuclear shell model is adopted to calculate the electric quadrupole moments for some Calcium isotopes $20 \mathrm{Ca}(N=21,23,25$, and 27) in the $f p$ shell. The wave function is generated using a two body effective interaction fpd6 and fp space model. The one body density matrix elements (OBDM) are calculated for these isotopes using the NuShellX@MSU code. The effect of the core-polarizations was taken through the theory microscopic by taking the set of the effective charges. The results for the quadrupole moments by using Bohr-Mottelson (B-M) effective charges are the best. The behavior of the form factors of some Calcium isotopes was studied by using Bohr-Mottelson (B-M) effective charges.
\end{abstract}

Key words: B-M effective charges, Form Factors, $f p$-shell, Nushell, Shell model.

\section{Introduction:}

The shell structure of single-particle levels in a spherical potential can be affected by the nuclear deformation (1). The first approximation representing closed core nuclei (the atomic number, $\mathrm{Z}$, is a magic number and, the neutron number is a magic number). Additionally, the outer-nucleons can move in a certain configuration space which interact with the core and each other by a residual interaction such particle-particle and particle-core interactions (2). These interactions depend on the selected model space and residual interactions; one can investigate this by comparing several experimental parameters such as spin/parity, excitation energy, the magnetic moment and the quadrupole moment and parametrization of the residual interaction and validity of the model space. The measurement of the quadrupole moment is often a good test to ascertain if the model space and the parameterizations are suitable (2). The nuclear electric quadrupole moment depends on a deflection of nuclear charge distribution from spherical symmetry which gives a useful measure of the core polarization especially if the valence nucleons are neutrons which do not directly participate to the electric quadrupole moment (3). Shell model calculations; adopt fixed measurement of the effective charges. The Bohr-Mottelson (B-M) (4) particle-vibration combines with model for the effective charges.

Department of Medical Physics, College of Applied Science, University of Fallujah, Al Anbar, Iraq.

E-mail: dr.ahmedphysics@uofallujah.edu.iq

*ORCID ID: https://orcid.org/0000-0002-1525-1406
Ogawa et al. (4) adopted the B-M which contain both the universal dependence $(N-Z) / A$ and the overcome effects for bound particles which showed that the experimental data of the quadrupole moments were in agreement (5).

The calculated ground-state electric quadrupole moments for the fpd6 and fpml3 interactions are compared with experimental values (for nuclei 41,43,44,45Sc, 41,43,45,47,49Ca, 45Ti). Some values calculated with the modified Kuo-Brown interaction of McGrory are also included. The effect of variations in the effective charges on the calculated moments is generally much larger than using different modified versions of the Kuo-Brown interaction. (6).

In the present work, we will adopt shell model calculations with a harmonic oscillator (HO) single-particle wave functions to calculate the quadrupole moments of some Calcium isotopes in the $f p$ shell. The calculations adopted different effective charges and were compared with experimental data. Also, the behavior of the form factors for some calcium isotopes by using BohrMottelson (B-M) effective charges is studied.

Theory

The electric quadrupole moment is associated with deformation of nuclei. The nuclear charge distribution in state $|J M\rangle$ is given by (7) $\rho_{J M}^{e}(r)=\left\langle J M\left|\sum_{k=1}^{A} e(k) \delta(r-r(k))\right| J M\right\rangle \ldots 1$ where $\rho_{J M}^{e}(r)$ describes the nuclear charge distribution, $e(k)$ denotes the charge of nucleon 
numbered $k$ and is axial symmetric about the z-axis. Therefore one has also the relation

$Q_{\mathrm{xx}}=Q_{\mathrm{yy}}$

$Q_{\mathrm{zz}}=-2 Q_{\mathrm{xx}}=-2 Q_{\mathrm{yy}}$

$Q_{\mathrm{xy}}=Q_{\mathrm{yz}}=Q_{\mathrm{x}} \mathrm{z}=0$

Hence, due to eq. above, only one independent quadrupole component, i.e. $Q_{z z}$ remains, which can be represented by

$Q(J M)=\int \rho_{J M}^{e}(r)\left(3 z^{2}-r^{2}\right) d r \quad \ldots 2$

when, alternatively, the quadrupole moment $Q(J M)$ is written in terms of the five spherical harmonics $\mathrm{Y}_{2 \mathrm{M}}(\hat{r})$ with $\mathrm{M}=0, \pm 1, \pm 2$, then, due to the axial symmetry of the charge distribution, the components with $\mathrm{M} \neq 0$ vanish. The connection between $Y_{20}(\hat{r})$ and Cartesian coordinates are given by:

$r^{2} Y_{20}(\hat{r})=\sqrt{\frac{5}{16 \pi}}\left(2 z^{2}-x^{2}-y^{2}\right)=$

$\sqrt{\frac{5}{16 \pi}}\left(3 z^{2}-r^{2}\right) \quad \ldots 3$

combining Eqs. 1, 2 and 3 one obtains:

$Q(J M)=\sqrt{\frac{16 \pi}{5}} \sum_{k=1}^{A} e(k)\left\langle J M\left|r^{2}(k) Y_{20}(\hat{r}(k))\right| J M\right\rangle$ 4

where $e(k)$ denotes the charge of nucleon numbered $k$, where $e(\mathrm{k})=0$ for a neutron and $e(k)=e$ for a proton.

Here also, a numerical factor $\sqrt{16 \pi / 5}$ occurs, because the quadrupole moment of an axially symmetric body is conventionally defined as $\left\langle 3 z^{2}-r^{2}\right\rangle$.

The expectation value in Eq. 4 still depends on the projection quantum number $M$.

For the given $J$ the moments for different values M are not independent, however, since they are defined by Clebsch-Gordan coefficients according to the Wigner-Eckart theorem. In nuclear physics the quadrupole moment of a state of angular momentum $J$ is defined as the expectation value of the state $M=J$. It is referred to as the spectroscopic, or static, quadrupole moment given by the definition:

$Q(J, M=J)=$

$\sqrt{\frac{16 \pi}{5}} \sqrt{\frac{J(2 J-1)}{(J+1)(2 J+1)(2 J+3)}}\langle J\|\hat{O}(E 2)\| J\rangle, \quad \ldots 5$

where the electric transition operator $\hat{O}(E 2)$ is defined as

$O(E L M)=\sum_{k=1}^{A} r^{L}(k) Y_{L M}(\hat{r}(k)) \quad \ldots \quad 6$

For the calculation of the quadrupole moment initial and final state wave function must be identical. The electric $2^{\mathrm{L}}$ pole operator $r^{L} Y_{L M}(\hat{r})$ possesses parity $(-1)^{\mathrm{L}}$, and the electric $2^{\mathrm{L}}$ pole moment should vanish for odd values of $L$. The intrinsic is defined with respect to the axis of symmetry of the charge distribution. The nuclear matrix element of the electromagnetic operators $\langle J\|\hat{O}(E 2)\| J\rangle$ between final nuclear states $\left(J_{f}\right)$ and the initial state $\left(J_{i}\right)$, where multipolarity $\boldsymbol{\tau}$ is the sum of the products of single-particle matrix elements times OBDM

$\left\langle J_{f}\|\hat{O}(\tau)\| J_{i}\right\rangle=$

$\sum_{j j^{\prime}} O B D M\left(J_{i}, J_{f}, j, j^{\prime}, \tau\right)\left\langle j^{\prime}\left\|\hat{O}_{J}(\tau)\right\| j\right\rangle \quad \ldots 7$

where the single-particle states represented

by $j^{\prime}$ and $j$ for the shell model space.

The model space matrix elements and sharing the effective charges $\left(e^{e f f}\left(t_{z}\right)\right)$, can be written by the electric matrix element

$M(E J)=\sum_{t_{z}} e^{e f f}\left(t_{z}\right)\left\langle J_{f}\left\|\hat{O}_{2}\left(\vec{r}, t_{z}\right)\right\| J_{i}\right\rangle_{M S} \ldots 8$

The formulated of the effective charges are written as (8)

$e^{e f f}\left(t_{z}\right)=e\left(t_{z}\right)+e \delta e\left(t_{z}\right), \delta e\left(t_{z}\right)=Z / A-$ $0.32(N-Z) / A-2 t_{Z}[0.32-0.3(N-Z) / A] \ldots 9$

The quadrupole moment in state $\mid J=2 M=0>$ for $J_{i}=J_{f}$ can be described by (7):

$Q(J=2)=\left(\begin{array}{ccc}J_{i} & J & J_{i} \\ -J_{i} & 0 & J_{i}\end{array}\right) \sqrt{\frac{16 \pi}{5}}\langle J \| \hat{O}(E 2)|| J\rangle=$

$\left(\begin{array}{ccc}J_{i} & 2 & J_{i} \\ -J_{i} & 0 & J_{i}\end{array}\right) \sqrt{\frac{16 \pi}{5}} M(E J) \quad \ldots 10$

the form factor between nuclear shell model states of final state $\left(J_{f}\right)$ and initial state $\left(J_{i}\right)$ involves the momentum transfer $(q)$ and the angular momentum $(\tau)$, between nuclear shell model states of final state $\left(J_{f}\right)$ and initial state $\left(J_{i}\right)$, is given by $(5,9)$

$|F(\eta \tau, q)|^{2}=$

$\frac{4 \pi}{Z^{2}(2 J+1)}\left|\left\langle J_{f} \| \hat{T}(\eta \tau, q)|| J_{i}\right\rangle F_{c . m}(q) F_{f . s}(q)\right|^{2} \quad \ldots 11$

where $\eta$ represents the longitudinal scattering. The correction for the lack of translational invariance in the shell model is $F_{c . m}=e^{q^{2} b^{2} / 4 A}$ and the finite size (f.s.) nucleon form factor is $F_{f . s}=[1+$ $\left.\left(\frac{q}{4.33}\right)^{2}\right]^{-2}$. The mass number represented by $\boldsymbol{A}$, and the harmonic oscillator (HO) length parameter represented by $\boldsymbol{b}$.

\section{Result and Discussion:}

Theoretical values of the electric quadrupole moments for $f p$-shell states, calculated as described, are presented and compared with experimental values as Tables. Results with three different effective charges, are given to illustrate the variations which can arise.

Calculations of the shell model are adopted using NuShellX@MSU code (10) to obtain excitation energy levels and the one body matrix elements (OBME). in the present work, The effective interaction $f p d 6$ (6) and model space $f p$ are used. The single particle-matrix elements can be calculated by size parameters $b$ and the HO. The 
size parameters $\boldsymbol{b}$ of some $\mathrm{Ca}$ isotopes are calculated with a mass number $(\mathrm{A})$ :

$b=\sqrt{\frac{\hbar}{M_{P} \omega}}$, where $\hbar \omega=45 A^{-1 / 3}-25 A^{-2 / 3}$ ( $M p=$ mass of proton) (11).

\section{Electric quadrupole moment}

The present work studies the microscopic structure of Ca nucleus in the fp shell. Calculations of the shell model should be a restricted model space where $f p$-model space adopted in the present work. Effective charges which are introduced in evaluating quadrupole moments in shell model are related to nucleon excitations in nuclei. Effective charges are important because of the polarization of the core which is not involved in the model space calculations. One set of effective charges, the B-M (8) are calculated according to Eq. 9 for some isotopes in the present work and shown in Table 1. For the two sets of effective charges, the standard effective charges (ST) are $e_{p}=1.36 e$ and $e_{n}=0.45$ $e$ (12). Lastly, for the three sets of effective charges, conventional effective charges (Con) are $e p=1.3 e$ and $e n=0.5 e$ (13). The nuclei containing $f p$-shell are supposed as an inert core ${ }^{40} \mathrm{Ca}$ and the valence nucleons (core outer nucleons) are distributed in $1 f_{7 / 2}, 2 p_{3 / 2}, \quad 1 f_{5 / 2}$ and $2 p_{1 / 2}$ orbits. The electric quadrupole moment (E2) were calculated with $f p d 6$ interaction (6) for 20Ca $(N=21,23,25$ and 27) isotopes with Eq.10 and the results were compared with experimental values in Ref. $(14,15)$ as shown in Tab 1and Fig.1.

In Fig. 1 the electric quadrupole moments for $20 \mathrm{Ca}$ isotopes are calculated with effective charges B-M (8). The calculated value of $Q(7 / 2$, $41 \mathrm{Ca}=-9 e \mathrm{fm}^{2}$ is in agreement with the experimental value (14) and shape nucleus of $41 \mathrm{Ca}$ is oblate where $\mathrm{B}-\mathrm{M}$ effective charges $1.17 e$ and $0.79 e$, for the proton and neutron, respectively. The calculated value of $Q(7 / 2,43 \mathrm{Ca})=-3.5 e \mathrm{fm}^{2}$ is overestimated with experimental values (14). The $\mathrm{B}-\mathrm{M}$ effective charges are $1.13 e$ and $0.72 e$, for the proton and neutron, respectively. The calculated value of $Q(7 / 2,45 \mathrm{Ca})=3.41 \mathrm{e} \mathrm{fm}^{2}$ is in close agreement with the experimental value (14). This value shows a prolate deformation which number of neutrons are odd $(N=25)$ and have positive parity, where $\mathrm{B}-\mathrm{M}$ effective charges are $1.12 e$ and $0.7 e$, for the proton and neutron, respectively. Finally, the calculated of value of $Q(7 / 2,47 \mathrm{Ca})=10.66 \mathrm{e} \mathrm{fm}^{2}$ is overestimated with experimental values (15) . This value shows a large prolate deformation for $N$ $=27$ where 20 neutrons in sd shell (core) and neutrons 7 neutrons in $1 f_{7 / 2}$ orbit and adopted the B$\mathrm{M}$ effective charges are $1.1 e$ and $0.66 e$, for the proton and neutron, respectively. These calculations are presented in Tab1 as shown in Fig.1.

Table1. Comparison of computed quadrupole moments in units of $e \mathrm{fm}^{2}$ with experimental values $(14,15)$ deduced from Bohr-Mottelson effective charges (B-M) (8) calculated and interaction $f p d 6(6)$.

\begin{tabular}{cccccccc}
\hline${ }_{20} \mathrm{Ca} N, A$ & $J^{\pi}$ & $b(f m)$ & $\begin{array}{c}\left(E_{X}\right)_{\exp } \\
M e V\end{array}$ & $\begin{array}{c}(\mathrm{EX})_{\mathrm{Theo}} \\
\mathrm{MeV}\end{array}$ & $\begin{array}{c}\mathrm{B}-\mathrm{M} \\
e_{p}, e_{n}\end{array}$ & $Q_{B-M}\left(\mathrm{efm}^{2}\right)$ & $\boldsymbol{Q}_{\text {exp }}\left(\mathrm{efm}^{2}\right)$ \\
\hline 21,41 & $7 / 2^{-}$ & 1.94 & 0 & 0 & $1.17,0.79$ & -9 & $-9 \pm 2^{(\mathrm{a})}$ \\
23,43 & $7 / 2^{-}$stable & 1.96 & 0 & 0 & $1.14,0.74$ & -3.5 & $-5.5 \pm 1^{(\mathrm{a})}$ \\
25,45 & $7 / 2^{-}$ & 1.972 & 0 & 0 & $1.12,0.7$ & 3.41 & $3.8 \pm 12^{(\mathrm{a})}$ \\
27,47 & $7 / 2^{-}$ & 1.983 & 0 & 0 & $1.1,0.66$ & 10.66 & $2.1 \pm 4^{(\mathrm{b})}$ \\
\hline
\end{tabular}

(a) Ref. the experimental values (14)

(b) Ref. the experimental values (15) 


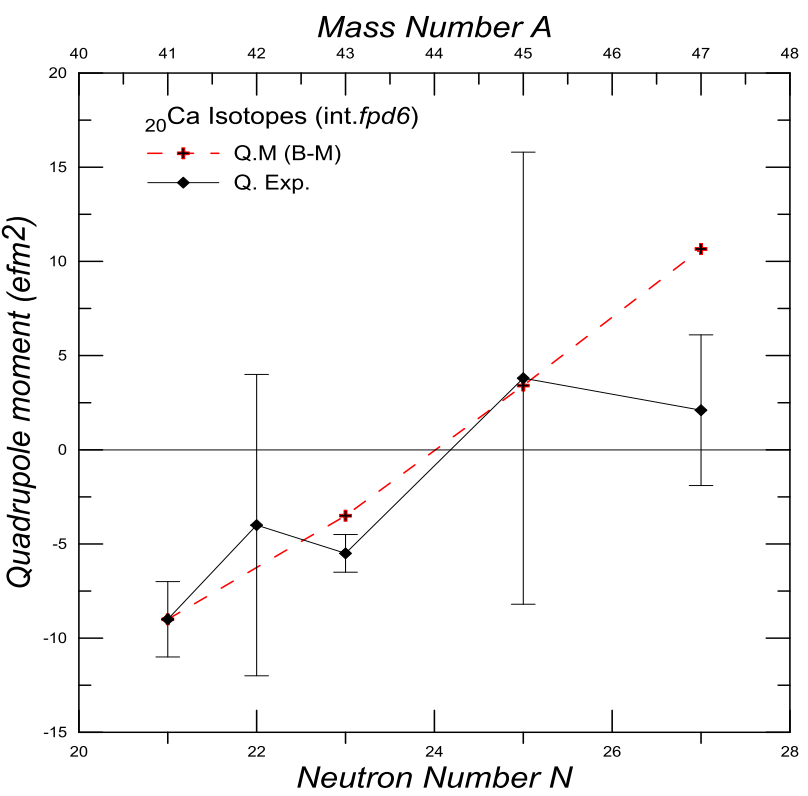

Figure 1. Quadrupole moments $\left(e \mathrm{fm}^{2}\right)$ calculated for some $\mathrm{Ca}$ isotopes by using the $\mathrm{B}-\mathrm{M}$ effective charges (8), the experimental values and results are compared $(14,15)$.
In Fig. 2 the electric quadrupole moments for $20 \mathrm{Ca}$ isotopes are calculated with the standard effective charges ST $\left(e_{p}=1.36, e_{n}=0.45\right)$ (12) for all isotopes in the present work and the results are tabulated in Table 2. The calculated value of $Q$ $(7 / 2,41 \mathrm{Ca})=-5.11$ e $\mathrm{fm}^{2}$ is overestimated with the experimental value (14). This value shows that the nucleus deformation is oblate and there is one particle (neutron) in $1 f_{7 / 2}$ shell. The calculated value of $Q\left(7 / 2^{-}, 43 \mathrm{Ca}\right)=-2.1 \mathrm{e} \mathrm{fm}^{2}$ is overestimated with experimental values (14). This value shows the presence of the deformation nucleus is oblate and three-valance nucleons (neutron) in $1 f_{7 / 2}$ orbit. The calculated value of $Q(7 / 2,45 \mathrm{Ca})=2.21 \mathrm{e} \mathrm{fm}^{2}$ is in close agreement with the experimental values(14), which shows a nucleus deformation is prolate. Finally, the calculated of value of $Q(7 / 2,47 \mathrm{Ca})$ $=7.34 \mathrm{e} \mathrm{fm}^{2}$ is overestimated with experimental values (15). This value shows a large prolate deformation for neutrons 20 in $\boldsymbol{s} \boldsymbol{d}$ shell where are frozen and seven-valence neutrons in $1 f_{7 / 2}$-shell these values are presented in Tab 2 .

Table2. The quadrupole moments (with units $e \mathrm{fm}^{2}$ ) of calculated with HO potential for Calcium (Ca) isotopes $(Z=20)$. Experimental quadrupole moments are taken from Stone $(14,15)$. Quadrupole moments calculated with effective charges of effective charges ST for proton and neutron 1.36, 0.45 , respectively (12) and by using interaction $f p d 6(6)$.

\begin{tabular}{cccccccc}
\hline${ }_{20} \mathrm{Ca} N, A$ & $J^{\pi}$ & $b(f m)$ & $\begin{array}{c}\left(E_{X}\right) \exp \\
M e V\end{array}$ & $\begin{array}{c}\left(E_{X}\right)_{\text {Theo. }} \\
M e V\end{array}$ & $\begin{array}{c}\mathrm{ST} \\
e_{p}, e_{n}\end{array}$ & $Q_{\text {theo }}\left(\right.$ efm $\left.^{2}\right)$ & $\boldsymbol{Q}_{\text {exp }}\left(\right.$ efm $\left.{ }^{2}\right)$ \\
\hline 21,41 & $7 / 2^{-}$ & 1.94 & 0 & 0 & $1.36,0.45$ & -5.11 & $-9 \pm 2^{(\mathrm{a})}$ \\
23,43 & $7 / 2^{-}$stable & 1.96 & 0 & 0 & $1.36,0.45$ & -2.1 & $-5.5 \pm 1^{(\mathrm{a})}$ \\
25,45 & $7 / 2^{-}$ & 1.972 & 0 & 0 & $1.36,0.45$ & 2.21 & $3.8 \pm 12^{(\mathrm{a})}$ \\
27,47 & $7 / 2^{-}$ & 1.983 & 0 & 0 & $1.36,0.45$ & 7.34 & $2.1 \pm 4^{(\mathrm{b})}$ \\
\hline
\end{tabular}

(a) Ref. the experimental values (14)

(b) Ref. the experimental values (15)

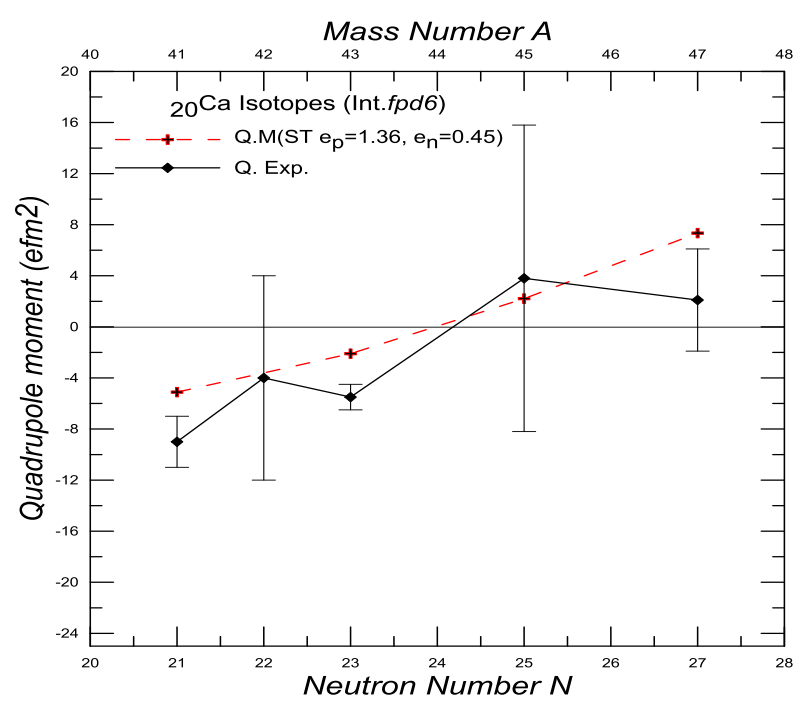

Figure 2. Quadrupole moments $\left(\mathrm{efm}^{2}\right)$ calculated for some Ca isotopes by using the ST effective charges $(12)$, the experimental values $(14,15)$ and results are compared.
In Fig.3 the electric quadrupole moments for $20 \mathrm{Ca}$ isotopes are calculated with conventional effective charges (Con) are $e_{p}=1.3 e$ and $e_{n}=0.5 e$ (13) for all isotopes in the present work. The calculated value of $Q(7 / 2,41 \mathrm{Ca})=-5.68 \mathrm{efm}^{2}$ is overestimated with the experimental value (14). This value shows deformation nucleus is oblate and one particle (neutron) in $1 f_{7 / 2}$ shell. The calculated value of $Q(7 / 2,43 \mathrm{Ca})=-2.37 \mathrm{efm}^{2}$ is overestimated with experimental values (14). This value shows deformation nucleus is oblate and three-valance nucleons (neutron) in $1 f_{7 / 2}$ orbit. The calculated value of $Q(7 / 2,45 \mathrm{Ca})=2.45 \mathrm{efm}^{2}$ is in close agreement with the experimental value (14). This value shows a nucleus deformation is prolate. Finally the calculated of value of $Q\left(7 / 2^{-}, 47 \mathrm{Ca}\right)=$ $8.16 \mathrm{efm}^{2}(15)$ is overestimated with experimental values (15). This value shows a large prolate deformation for neutrons 20 in $\boldsymbol{s} \boldsymbol{d}$ shell as frozen and neutrons 7 in $1 f_{7 / 2}$ shell. These results are presented in Tab 3. 
Table3. Comparison of the computed quadrupole moments (with units $\mathrm{efm}^{2}$ ) with experimental values (14, 15) deduced from conventional effective charges effective charges (Con) for proton and neutron1.3, 0.5, respectively (13) and by using interaction $f p d 6(6)$.

\begin{tabular}{cccccccc}
\hline${ }_{20} \mathrm{Ca}$ & $J^{\pi}$ & $b(f m)$ & $\begin{array}{c}\left(E_{X}\right) \exp \\
\mathrm{MeV}\end{array}$ & $\begin{array}{c}\left(E_{X}\right)_{\text {Theo. }} \\
\mathrm{MeV}\end{array}$ & $\begin{array}{c}\text { Con } \\
e_{p}, e_{n}\end{array}$ & $\begin{array}{c}Q_{\text {con }} \\
\left(\mathrm{efm}^{2}\right)\end{array}$ & $\begin{array}{c}\boldsymbol{Q}_{\exp } \\
\left(\mathrm{efm} \mathrm{fm}^{2}\right)\end{array}$ \\
\hline 21,41 & $7 / 2^{-}$ & 1.94 & 0 & 0 & $1.3,0.5$ & -5.68 & $-9 \pm 2^{(\mathrm{a})}$ \\
23,43 & $7 / 2^{-}$stable & 1.96 & 0 & 0 & $1.3,0.5$ & -2.37 & $-5.5 \pm 1^{(\mathrm{a})}$ \\
25,45 & $7 / 2^{-}$ & 1.972 & 0 & 0 & $1.3,0.5$ & 2.45 & $3.8 \pm 12^{\text {(a) }}$ \\
27,47 & $7 / 2^{-}$ & 1.983 & 0 & 0 & $1.3,0.5$ & 8.16 & $2.1 \pm 4^{(\mathrm{b})}$ \\
\hline
\end{tabular}

(a) Ref. the experimental values (14)

(b) Ref. the experimental values (15)

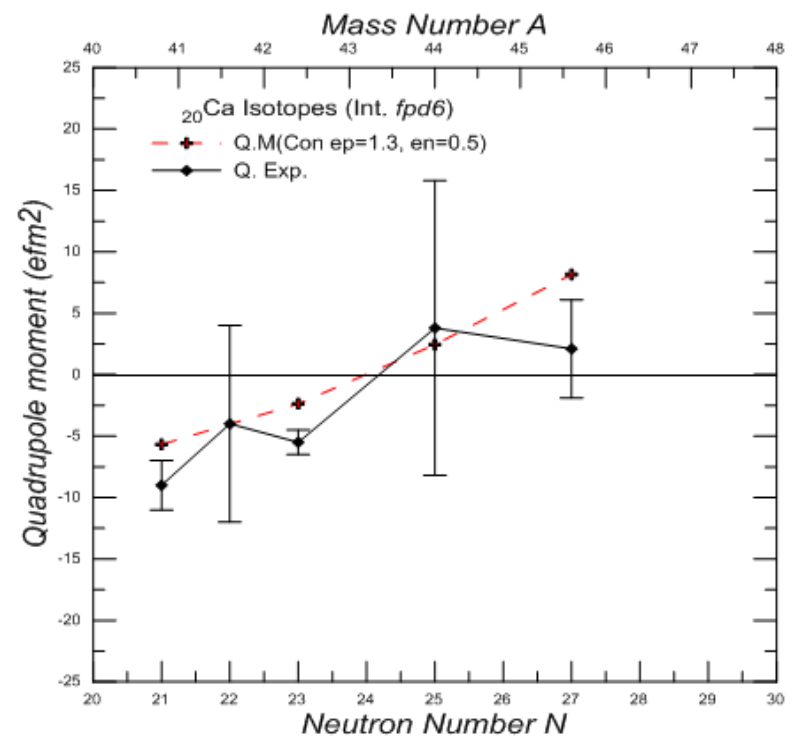

Figure 3. Quadrupole moments $\left(e \mathrm{fm}^{2}\right)$ calculated for some $\mathrm{Ca}$ isotopes by using conventional effective charges effective charges (Con) $(13)$, the experimental values $(14,15)$ and results are compared.

In the Fig.4 comparison of experimental values and calculated quadrupole moments (theoretical) are shown for $\mathrm{Ca}$ isotopes considered in this work where the line represents the experimental values on the $\mathrm{X}$-axis are equal to the theoretical values calculated on the $\mathrm{Y}$-axis $\left(Q_{\exp }=\right.$ $\left.Q_{\text {theo }}\right)$. From all the Figures presented, good agreement is obtained with the experimental values for most of the isotopes while it deteriorates for some others where they need further investigation.

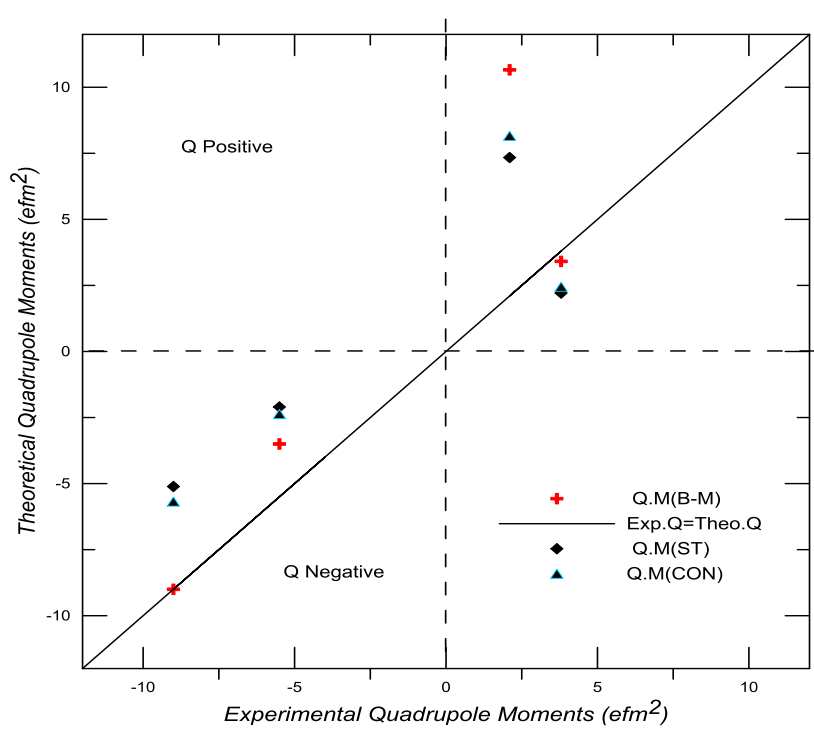

Figure 4. Comparison of experimental values and calculated quadrupole moments for some $\mathrm{Ca}$ isotopes by using different the effective charges.

\section{The Form Factors}

The calculations of the form factors for some $\mathrm{Ca}$ isotopes where no experimental data are available, the $f p$ model space with the interaction fpd6 (6) was adopted. The model space matrix elements are obtained using Eq.8, where the required one-body density matrix was calculated using code the NuShellX@MSU (10). The form factors $\mathrm{C} 2$ is calculated using an expression of the transition charge density operator from Eq.11.

The electric-charge form factors are computed for $41,43,45 \mathrm{Ca}$ isotopes as the Fourier transform of the charge density. The present work calculated $|\mathrm{F}(\mathrm{q})|^{2}$, where the form factors for $41 \mathrm{Ca}$ and for $43 \mathrm{Ca}$ are shown in Fig.5 and form factor for $45 \mathrm{Ca}$ is shown in Fig.6. Calculation of the form factors is done by using set effective charges, the first conventional effective charges $\left(e_{p}=1.3, e_{n}=0.5\right)$, the second B-M effective charges and the third ST effective charges $\left(e_{p}=1.36, e_{n}=0.45\right)$. These Figures were the highest form factor values by using effective charges B-M and the experimental values unavailable for comparison. 

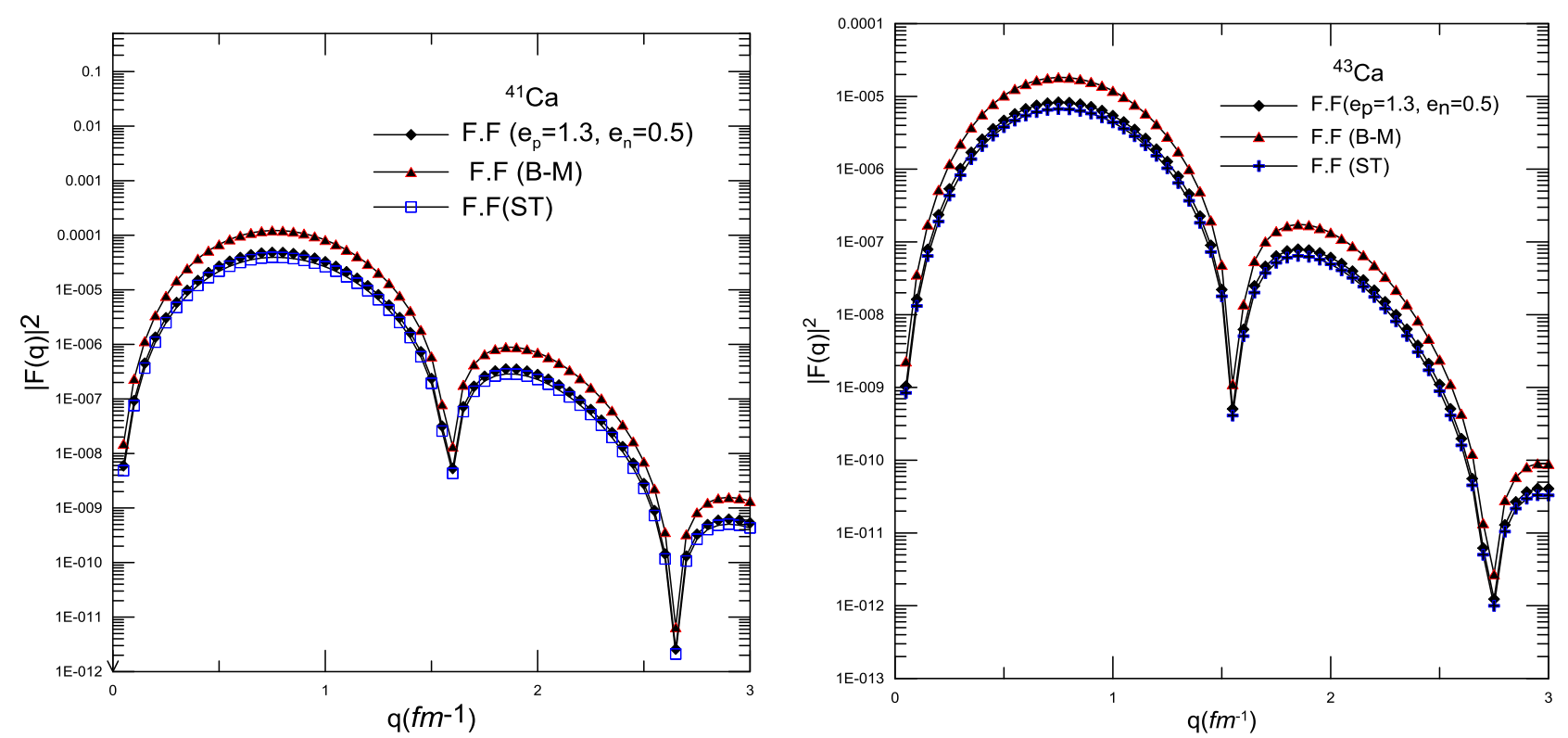

Figure 5. Squared charge form factor for $41 \mathrm{Ca}$ and $43 \mathrm{Ca}$ as a function of the momentum transfer (q). It has been obtained from Eq. 11, and by using the different effective charges.

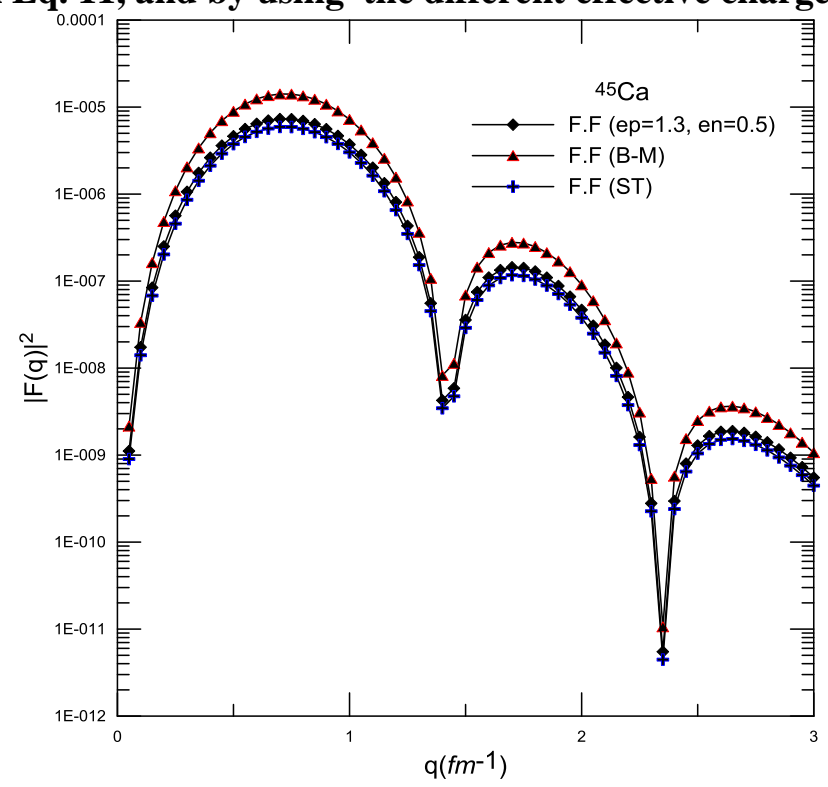

Figure 6. Squared charge form factor for $45 \mathrm{Ca}$ as a function of the momentum transfer (q). It has been obtained from Eq. 11, and by using the different effective charges.

\section{Conclusion:}

The characteristics of the quadrupole collectivity that develops $N \geq 20$ are discussed by comparing the experimental values of the quadrupole moments with the shell model calculations. Shell model is adopted to calculate the quadrupole moments of $\mathrm{Ca}(41,43,45$, and 47). Our calculations include core-polarization effects through three effective charges. The set of effective charges are Bohr-Mottelson (B-M), standard effective charges (ST) and conventional effective charges (Con). The results of the quadrupole moment by using fpd6 interaction are in agreement with the experiment when using B-M effective charges. In the case, the results almost agree with the experimental value. The results show an increase of the quadrupole moments as increasing the neutron number. Also, the results show an increase in the values of the form factors using the effect of core polarization is found essential in both the momentum transfer and transition strengths dependence and gives a good description for the behavior of charge distribution.

\section{Author's declaration:}

- Conflicts of Interest: None.

- I hereby confirm that all the Figures and Tables in the manuscript are mine. Besides, the Figures and images, which are not mine, have been given the permission for re-publication attached with the manuscript. 
- Ethical Clearance: The project was approved by the local ethical committee in University of Fallujah.

\section{References:}

1. Hadyńska-Kl K, Napiorkowski P J, Zielińska M, Srebrny J, Maj A, Azaiez F et al. Quadrupole collectivity in 42Ca from low-energy Coulomb excitation with AGATA. Phys. Rev. C 2018; 97: 024326.

2. Neyens G. Nuclear magnetic and quadrupole moments for nuclear structure research on exotic nuclei. Reports Prog. Phys. 2003; 6(4): 633-689.

3. Radhi R A, Ali A H. Microscopic effective charges and quadrupole moments of sd-shell and sd-pf crossshell nuclei with $Z \geq 13$. Iraqi J. Phys. 2016; 14(30): 136-149.

4. Ogawa H, Asahi K, Sakai K, Suzuki T, Izumi H, Miyoshi $\mathrm{H}$, et al. Electric quadrupole moment of [Formula Presented] and anomalous effective charges for neutron-rich nuclei. Phys. Rev. C. 2003; 67(6): 9.

5. Radhi R A, Alzubadi A A, Ali A H. Magnetic dipole moments, electric quadrupole moments, and electron scattering form factors of neutron-rich sd-pf crossshell nuclei. Phys. Rev. C. 2018; 97(6): 1-13.

6. Richter WA, Van der Merwe MG, Julies RE, Brown BA. New effective interactions for the Oflp shell. Nucl. Physics, Sect. A 1991;523: 325-353.
7. Brussard P, Glaudemans P. Shell-Model Applications in Nuclear Spectroscopy. North Holland. Amesterdam. 1977; 452.

8. Ahmed H A. Study of the Electric Quadrupole Moments for some Scandium Isotopes Using Shell Model Calculations with Different Interactions. B S J 2019; 15(3): 304-309.

9. Ban S. The Nuclear Structure for Exotic NeutronRich of ${ }^{42,43,45,47}$ K Nuclei. B S J. 2016; 13.

10. Brown B. A, W. D. M. Rae. The Shell-Model Code NuShellX@MSU. Nucl. Data Sheets 2014; 120: 115118.

11. Ahmed H A, Akram M A. Reduced Quadruple Transition Probability B(E2) for Some Oxygen Isotopes Using Different Interactions and Effective Charges. IOP Conf. Series: J. Phys. 2018; 1032.

12. Richter WA, Mkhize S, Brown BA. Sd-shell observables for the USDA and USDB Hamiltonians. Phys. Rev. C. 2008; 78(6): 064302.

13. Radhi R A, Alzubadi A A, Ali AH. Calculations of the Quadrupole Moments for Some Nitrogen Isotopes in $p$ and $p s d$ Shell Model Spaces Using Different Effective Charges. Iraqi J. Sci. 2017; 58(2): 878883.

14. Stone J. Table of Nuclear Electric Quadrupole Moments. At. Data Nucl. Data Tables; 2016; 112: 28.

15. Stone J. Table of nuclear magnetic dipole and electric quadrupole moments. At. Data Nucl. Data Tables; 2005;90: 75-176.

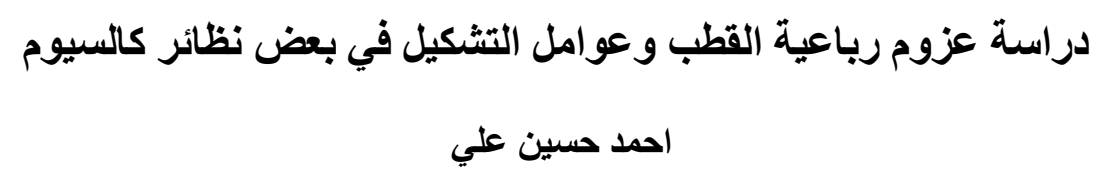

قسم الفيزياء الطبية، كلية العلوم التطبيقية، جامعة الفلوجة، الانبار، العراق.

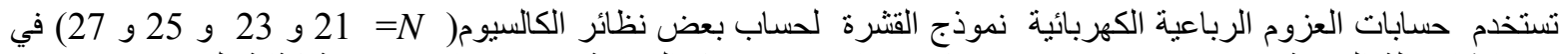

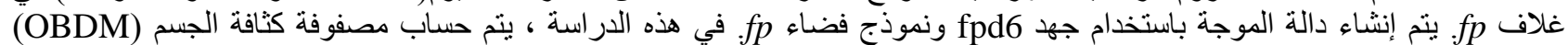

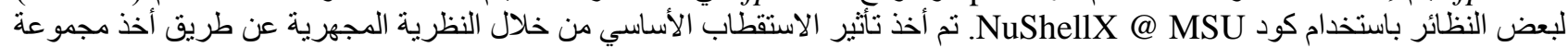

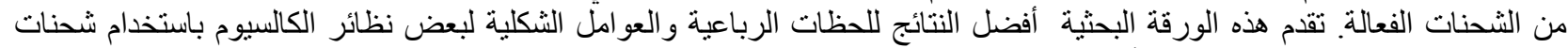
Bohr-Mottelson (B- الفعالة. أيضا ، در اسة سلوك عو امل الثكل لبعض نظائر الكالسيوم باستخدام شحنات (Bohr-Mottelson) B-M (M ) الفعالة.

الكلمات المفتاحية: شحنات بور موتلسن الفعالة، عو امل التشكيل، غلاف NuShellX،fp ، نموذج الاغلفة. 